\title{
Gold catalysis in organic synthesis: efficient intramolecular cyclization of $\gamma$-acetylenic carboxylic acids to 5-exo-alkylidene-butyrolactones
}

\author{
Emilie Genin, Patrick Y. Toullec, Peggy Marie, Sylvain Antoniotti, Célia Brancour, \\ Jean-Pierre Genêt,* and Véronique Michelet*
}

Laboratoire de Synthèse Sélective Organique et Produits Naturels, E.N.S.C.P., UMR 7573, 11 rue P. et M. Curie, F-75231 Paris Cedex 05, France

E-mail: jean-pierre-genet@enscp.fr, veronique-michelet@enscp.fr

Dedicated to Professor Lutz Tietze on the occasion of his $65^{\text {th }}$ birthday

\begin{abstract}
We have found that functionalized acetylenic acids may be cyclized under extremely mild conditions, at room temperature in the presence of $\mathrm{AuCl}$ catalyst and without the use of additives. The corresponding 5-exo-alkylidene-butyrolactones were isolated in high yields, and this process constitutes an easy and efficient route to highly valuable building blocks of natural products having biological interest.
\end{abstract}

Keywords: Gold, catalysis, cyclization, carboxylic acids, functionalized $\gamma$-butyrolactones

\section{Introduction}

Recent years have witnessed tremendous growth in the number of gold-catalyzed highly selective chemical transformations. ${ }^{1}$ Gold was considered as an inert metal for a long time, and its ability to behave as a soft Lewis-acid has recently been recognized as a source of inspiration for organic chemists. Gold catalysts may activate unsaturated functionalities such as alkenes, allenes and alkynes, and therefore allow the creation of carbon-carbon and carbon-heteroatom bonds under extremely mild conditions. ${ }^{1}$ Thus the addition of various nucleophiles to alkynes offers a fascinating opportunity to build up several complex and/or cyclic molecules. Various internal nucleophiles such as alkenyl, ${ }^{2}$ sulfur- ${ }^{3}$ oxygen- ${ }^{4}$ and nitrogen- ${ }^{5}$ containing functions have been used in this regard. As part of our ongoing studies on metal-catalyzed atomeconomical reactions, ${ }^{6}$ we have been interested in the use of gold for simple and highly efficient transformations. We have found that $\mathrm{Au}^{\mathrm{I}}$ catalyst promotes a highly efficient cycloisomerization of bis-homopropargylic diols under very mild conditions and in very short time. This process, 
starting from easily accessible materials, was shown to be general, leading to functionalized strained bicyclic ketals. ${ }^{6 c}$ In spite of the weaker nucleophilicity of the carboxylic function, the cyclization of acetylenic acids was also possible for the first time in the presence of gold. ${ }^{6 a}{ }^{7}$ The corresponding $\gamma$-lactones indeed constitute important building blocks for the synthesis of a myriad of natural or biologically active derivatives. ${ }^{8,9}$ We report herein our full investigations on cycloisomerizations of acetylenic acids leading to functionalized $\gamma$-butyrolactones.

\section{Results and Discussion}

Putting the stress on the preparation of acetylenic acids possessing functionalized side chains in a convenient and general way, we decided to take advantage of the malonic synthesis to introduce a variety of substituents at the carbon adjacent to the acid group (Scheme 1). We therefore synthesized some propargylic diesters starting from available 1: the alkylation step was performed under conventional conditions. The monosaponification reaction then occurred selectively in basic methanolic medium. ${ }^{10}$ The carboxylic acids 3a-c bearing an allylic or various substituted alkenyl side chains were obtained in moderate to excellent isolated yields. A bispropargylic derivative 2d was also synthesized and transformed into the monoacid $\mathbf{3 d}$ in high isolated yields. Starting from 1, we also envisaged the preparation of a halogen- containing derivative, which was achieved via an electrophilic chlorination reaction under mild conditions. The chloro- substituted carboxylic acid 3e was isolated in 59\% yield in two steps. An allylic free alcohol substrate was also easily prepared according to Trost's Pd-catalyzed methodology. ${ }^{11}$ The alkyne $\mathbf{3 f}$ was obtained as an $E / Z$ mixture in $53 \%$ yield.

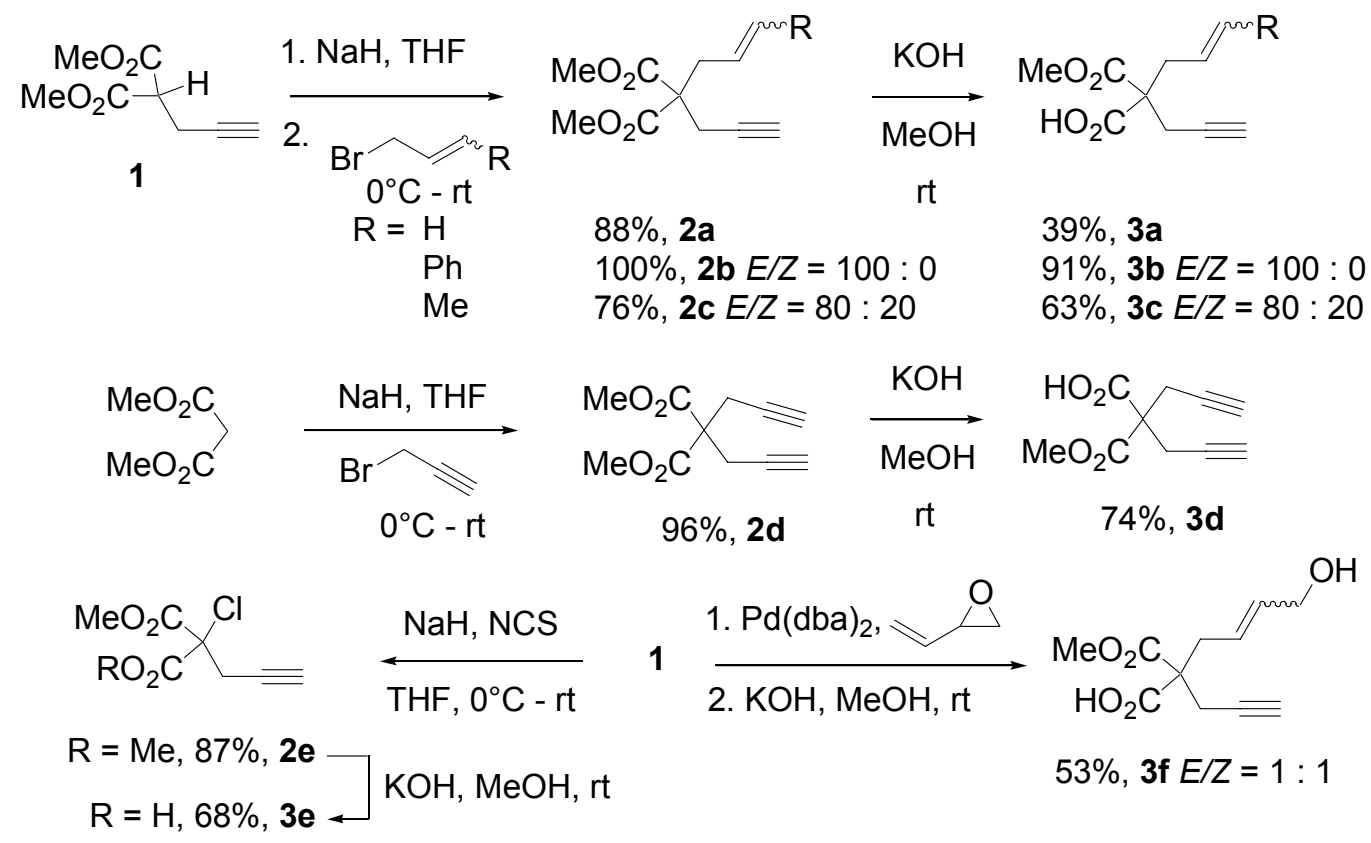

\section{Scheme 1}


Some other derivatives were prepared starting from the commercially available substituted diesters 4 and $\mathbf{5}$ (Scheme 2). More specifically, the propargylic moiety was introduced efficiently to $n$-butyl and benzyl substituted malonates in $90-93 \%$ yield. In order to avoid transesterification, monosaponification steps were performed this time in ethanol and afforded the corresponding acids $\mathbf{3 g}-\mathbf{h}$ in good yields. In the case of the $n$-butyl diester $\mathbf{2} \mathbf{h}$, we decided to introduce an aromatic substitution, as these motifs are present in several lactonic natural products. $^{12}$ The aryl- substituted acids 3i-k were therefore obtained via a Sonogashira crosscoupling reaction ${ }^{13}$ in the presence of iodobenzene, 4-cyano-iodobenzene or 4-methoxyiodobenzene, followed by the classic mono- saponification step.
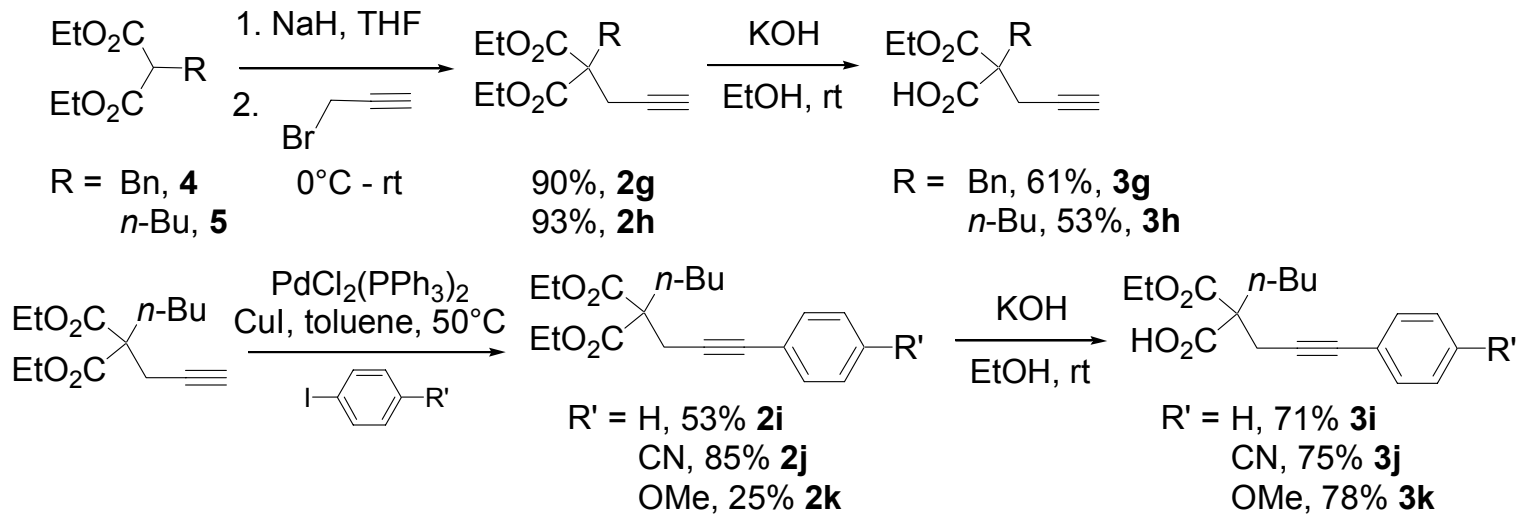

\section{Scheme 2}

Having in hand a large variety of acetylenic carboxylic acids 3a-k, we then performed a catalyst screening to optimize the cyclization conditions (Table 1). The standard substrate $\mathbf{3 b}$ was subjected to various gold catalysts and other activating agents in different solvents. The use of 5 mol.\% of $\mathrm{AuCl}$ in acetonitrile cleanly afforded in a short reaction time (2h) the corresponding 5exo-butyrolactone $\mathbf{6 b}$ in $90 \%$ yield at room temperature (entry 1).

The efficiency of gold(I) was compared with gold(III), silver triflate, and scandium triflate in acetonitrile (entries 2-4). It's noteworthy that the formation of lactone $\mathbf{6 b}$ was not possible in the presence of $\mathrm{HCl}$ in acetonitrile, as has been observed for Au-catalyzed cyclization reactions ${ }^{2 \mathrm{~h}}$ (entry 5).

Gold catalysts exhibited much better activation efficiency within 2 hours. Gold(I) was chosen for its easy handling ability and its lower sensitivity to moisture. The use of toluene, dichloromethane, or 1,2-dichloroethane led to the formation of the desired lactone in lower isolated yields (Table 1, entries 6-8). The versatility of $\mathrm{AuCl}$ was then evaluated for other functionalized acid derivatives (Table 2). 
Table 1. Cyclization of carboxylic acid $\mathbf{3 b}$

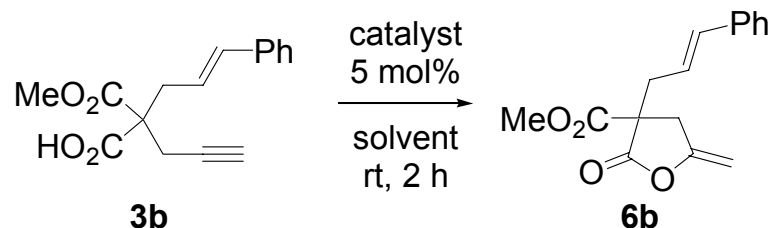

$3 b$

$6 b$

\begin{tabular}{cccc}
\hline Entry & Catalyst $^{\mathrm{a}}$ & Solvent & Yield $(\%)^{\mathrm{b}}$ \\
\hline 1 & $\mathrm{AuCl}$ & $\mathrm{CH}_{3} \mathrm{CN}$ & 90 \\
2 & $\mathrm{AuCl}_{3}$ & $\mathrm{CH}_{3} \mathrm{CN}$ & 84 \\
3 & $\mathrm{AgOTf}$ & $\mathrm{CH}_{3} \mathrm{CN}$ & $10^{\mathrm{c}}$ \\
4 & $\mathrm{Sc}(\mathrm{OTf})_{3}$ & $\mathrm{CH}_{3} \mathrm{CN}$ & 0 \\
5 & $\mathrm{HCl}$ & $\mathrm{CH}_{3} \mathrm{CN}$ & 0 \\
6 & $\mathrm{AuCl}$ & $\mathrm{Tolluene}$ & 60 \\
7 & $\mathrm{AuCl}$ & $\mathrm{CH}_{2} \mathrm{Cl}_{2}$ & 63 \\
8 & $\mathrm{AuCl}$ & $\mathrm{C}_{2} \mathrm{H}_{4} \mathrm{Cl}_{2}$ & 57 \\
\hline
\end{tabular}

${ }^{\mathrm{a}} 5 \mathrm{~mol} . \%$; $^{\mathrm{b}}$ isolated yield; ${ }^{\mathrm{c}}$ conversion

The presence of allylic or propargylic side chains was compatible with the reaction conditions and no other competitive addition to these unsaturated moieties ${ }^{7}$ was observed (Table 2 , entries 1,2). The chloro- or free alcohol- substituted carboxylic acids 3e-f underwent a clean and selective cyclization reaction leading to $\gamma$-lactones $\mathbf{6 e - f}$ in $85-95 \%$ isolated yields. The benzyl- and $n$-butyl- functionalized lactones $\mathbf{6 g - h}$ were also isolated in excellent yields.

The cyclizations delivered only the 5-exo product. The stereochemical outcome of the addition process on the alkyne moiety was established on substituted alkynyl derivatives. The aryl functionalized carboxylic acids 3i-k were subjected to the optimized conditions. We were pleased to see that the 5-exo process was still effective and the stereochemistry of the resulting alkenes was proved to be exclusively $Z$-. The stereochemistry of the double bond was demonstrated by performing NOESY NMR experiments on lactones $\mathbf{6 j}$ and $\mathbf{6 k}$. It has to be noted that the electronic effects influenced the yield of the reaction. An electron-withdrawing group such as a cyano group enhanced the reactivity of the carboxylic acid $\mathbf{3} \mathbf{j}$, whereas an electrondonating group such as a methoxy group deactivated the alkynyl moiety and therefore produced a lower yield for the cyclization reaction. 
Table 2. AuCl-catalyzed cyclization of acetylenic acids in acetonitrile at room temperature

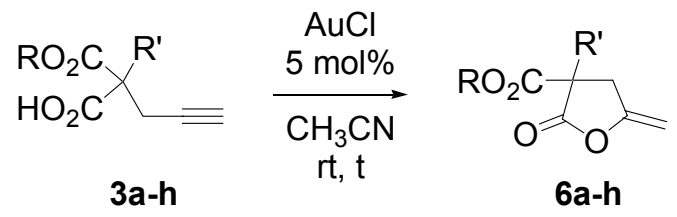

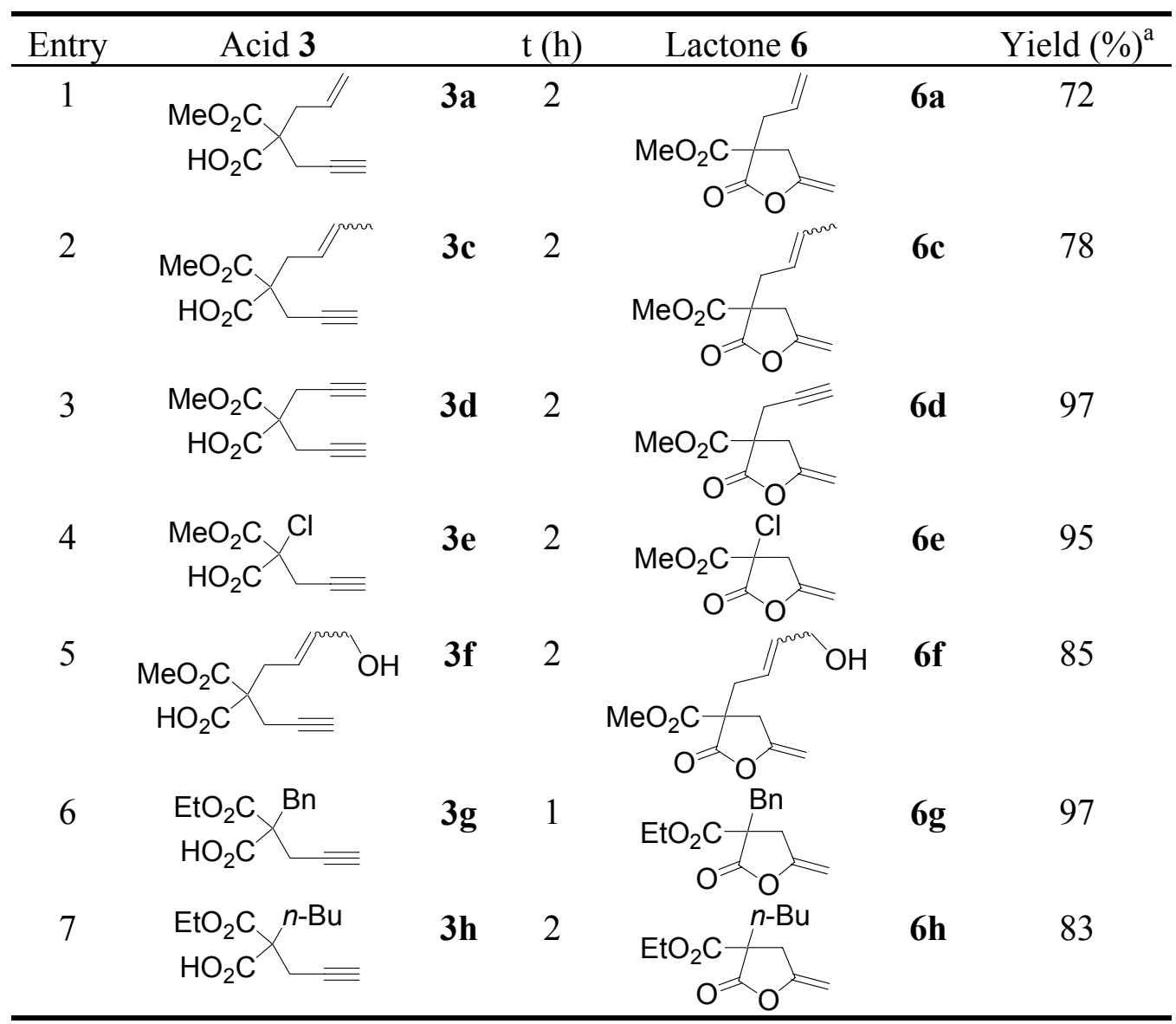

${ }^{a}$ isolated yield

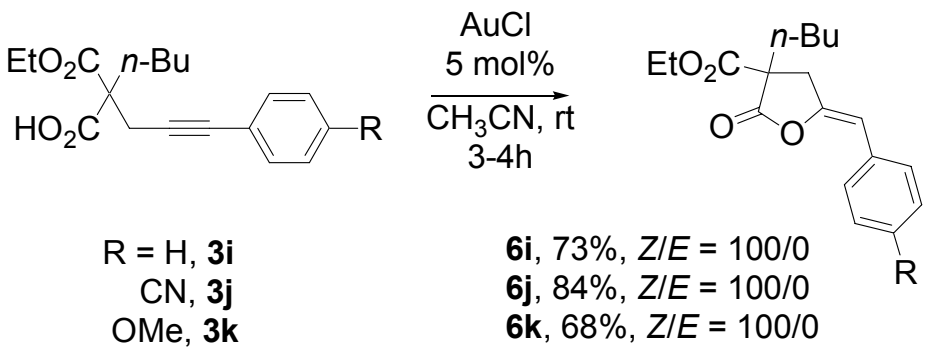

Scheme 3 
Thus, a tentative mechanism for the Au-catalyzed cyclization reaction is proposed in Scheme 4. The reaction may be initiated by the formation of the $\pi$-alkynyl complex $\mathbf{A}$ through the complexation of the unsaturated triple bond to the Au catalyst. The electrophilicity of the alkyne is therefore enhanced in the same manner as in the case of other metal-catalyzed nucleophilic addition reactions to unsaturated moieties. ${ }^{14}$ The addition of the carboxylic acid function to the triple bond then occurs anti- to the gold catalyst and leads to the vinylgold intermediate B. The last step may then be protodemetalation leading to the $\gamma$-lactone and regenerating the Au catalyst.

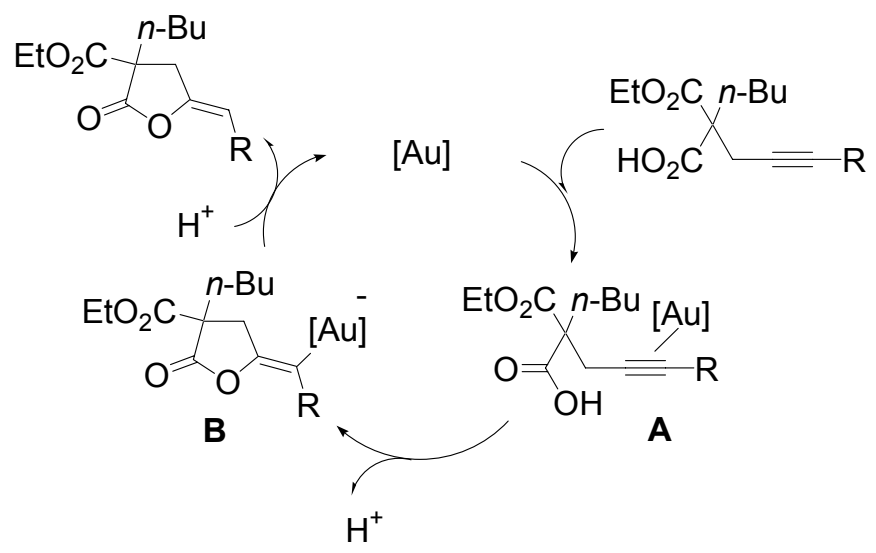

Scheme 4. Tentative mechanism for the Au-catalyzed cyclization reaction of acetylenic carboxylic acids.

In summary, a highly efficient cyclization reaction of $\gamma$-acetylenic carboxylic acids was developed by using $\mathrm{AuCl}$ as a simple commercially available catalyst in acetonitrile at room temperature. The carboxylic substrates were very easily prepared from simple precursors and the cyclization reactions afforded selectively the corresponding 5-exo-alkylidene-butyrolactones. The mechanism was investigated through the preparation and reaction of substituted alkynes. Further investigations will be dedicated to the synthesis of $\delta$-lactones, as well as the application in natural product synthesis.

\section{Experimental Procedures}

General Procedures. Reagents were commercially available from Acros, Aldrich or Avocado and were used without further purification. RT denotes room temperature. All manipulations involving air-sensitive reagents were carried out under argon and Schlenk techniques for catalytic tests. Column chromatography was performed with E. Merck 0.040-0.063 mm Art. 11567 silica gel. ${ }^{1} \mathrm{H}$ NMR and ${ }^{13} \mathrm{C}$ NMR were recorded on a Bruker AV 300 instrument. All signals for ${ }^{1} \mathrm{H}$ and ${ }^{13} \mathrm{C}$ NMR were expressed as ppm downfield from $\mathrm{Me}_{4} \mathrm{Si}$ used as an internal 
standard $(\delta)$. Coupling constants $(J)$ are reported in $\mathrm{Hz}$ and refer to apparent peak multiplicities. Mass spectrometry analyses were performed at the Ecole Nationale Supérieure de Chimie de Paris with a Hewlett-Packard HP 5989 A. Direct introduction experiments were done by electronic impact. High resolution mass spectra were performed on a Varian MAT311 instrument at the Ecole Normale Supérieure (Paris). The spectral characterizations of $\mathbf{2 a},{ }^{15} \mathbf{2 b},{ }^{15}$ $\mathbf{2 c},{ }^{16} \mathbf{2 d},{ }^{17} \mathbf{2} \mathbf{f},{ }^{11} \mathbf{2 g},{ }^{18} \mathbf{2 h},{ }^{19} \mathbf{6 a - 6 j}{ }^{6 \mathrm{a}}$ are identical to those published in the literature.

Standard procedure for alkylation reaction of malonates and synthesis of $\mathbf{2 a - d , ~} \mathbf{2 g}-\mathbf{h}$. Under an inert atmosphere (Ar) $\mathrm{NaH}$ (1.1 eq., $\mathrm{M}=24,60 \mathrm{wt} . \%$ in mineral oil) was added portionwise at $0^{\circ} \mathrm{C}$ to a solution of substrate ( 1 eq.) in anhydrous THF. The mixture was allowed to warm to RT, and the bromide (1.1 eq.) was slowly added. The mixture was stirred at RT to completion of the reaction, quenched with water, and extracted with $\mathrm{Et}_{2} \mathrm{O}$. The combined organic layers were washed with saturated aqueous $\mathrm{NaCl}$ solution, dried over $\mathrm{MgSO}_{4}$, filtered and concentrated under reduced pressure. The crude product was purified by silica gel flash chromatography (cyclohexane/EtOAc, 80/20 to 95/5) if necessary.

Standard procedure for monosaponification reaction. Under inert atmosphere (Ar) a solution of $\mathrm{KOH}$ (1.2 eq., $\mathrm{M}=56$ ) in anhydrous methanol or ethanol was added to a solution of substrate ( 1 eq). The mixture was stirred at RT to completion of the reaction. Solvent was then evaporated under reduced pressure and the crude solid was dissolved in $\mathrm{Et}_{2} \mathrm{O}$. The organic layer was washed three times with aqueous sodium bicarbonate ( $5 \mathrm{wt}$. \%). The aqueous phase was acidified to $\mathrm{pH}$ $=1$ with concentrated $\mathrm{HCl}$ and then extracted with $\mathrm{Et}_{2} \mathrm{O}$, dried over $\mathrm{MgSO}_{4}$, filtered, and concentrated under reduced pressure.

Standard procedure for Sonogashira reaction. Under an inert atmosphere ( $\mathrm{Ar}) \mathrm{PdCl}_{2}(5 \%$ eq., $M=177.3)$ and triphenylphosphane (10\% eq., $M=262.3)$ were added to a solution of aryl iodide (1.2 eq.) in anhydrous THF $(0.1 \mathrm{~mol} / \mathrm{L})$. The mixture was stirred at RT for $10 \mathrm{~min}$, then triethylamine ( 2 eq., $\mathrm{M}=101.19, \mathrm{~d}=0.726$ ) was added. The mixture was stirred at $\mathrm{RT}$ for 10 minutes more, then $\mathrm{CuI}(0.1$ eq., $\mathrm{M}=190.5)$ was added. After $30 \mathrm{~min}$ stirring, a solution of substrate (1 eq.) in anhydrous THF $(0.7 \mathrm{~mol} / \mathrm{L})$ was added and the mixture stirred at $50^{\circ} \mathrm{C}$ for 16 h. After completion of the reaction, solvent was evaporated under reduced pressure and the crude mixture was purified by silica gel flash chromatography (cyclohexane/EtOAc, 92/8) to give the desired product.

Standard procedure for acid cyclization. A mixture of acetylenic acid and $\mathrm{AuCl}(5 \mathrm{~mol} . \%)$ in degassed acetonitrile (1.2 mol. $\left.\mathrm{L}^{-1}\right)$ was stirred under an argon atmosphere at RT. After completion of the reaction, the mixture was filtered through a short pad of silica (EtOAc) and the solvents were evaporated under reduced pressure to give the corresponding lactone.

2-(Methoxycarbonyl)-2-(prop-2-ynyl)pent-4-enoic acid (3a). ${ }^{1} \mathrm{H}-\mathrm{NMR}\left(300 \mathrm{MHz}, \mathrm{CDCl}_{3}\right)$ : $\delta=2.05(\mathrm{t}, J=2.7 \mathrm{~Hz}, 1 \mathrm{H}), 2.75-2.84(\mathrm{~m}, 4 \mathrm{H}), 3.78(\mathrm{~s}, 3 \mathrm{H}), 5.16(\mathrm{~d}, J=10,0 \mathrm{~Hz}, 1 \mathrm{H}), 5.21(\mathrm{~d}, J$ $=18.0 \mathrm{~Hz}, 1 \mathrm{H}), 5.55-5.80(\mathrm{~m}, 1 \mathrm{H}) .{ }^{13} \mathrm{C}-\mathrm{NMR}\left(75 \mathrm{MHz}, \mathrm{CDCl}_{3}\right): \delta=22.9,36.8,53.0,57.0,71.8$, 78.4, 120.3, 131.2. CI-MS $\left(\mathrm{NH}_{3}\right) m / z 197(\mathrm{M}+\mathrm{H})^{+}, 214\left(\mathrm{M}+\mathrm{NH}_{4}\right)^{+}$. HRMS calculated for $\mathrm{C}_{10} \mathrm{H}_{12} \mathrm{O}_{4}$ : 197.0814, found: 197.0818 . 
(E)-2-(Methoxycarbonyl)-5-phenyl-2-(prop-2-ynyl)pent-4-enoic acid (3b). ${ }^{1} \mathrm{H}-\mathrm{NMR}$ (300 $\left.\mathrm{MHz} \mathrm{CDCl}_{3}\right): \delta=2.07(\mathrm{t}, J=2.7 \mathrm{~Hz}, 1 \mathrm{H}), 2.87(\mathrm{~d}, J=2.7 \mathrm{~Hz}, 2 \mathrm{H}), 2.99-2.94(\mathrm{~m}, 2 \mathrm{H}), 3.79$ (s, $3 \mathrm{H}), 6.11(\mathrm{dt}, J=15.7,7.8 \mathrm{~Hz}, 1 \mathrm{H}), 6.45(\mathrm{~d}, J=15.7 \mathrm{~Hz}, 1 \mathrm{H}), 7.35-7.25(\mathrm{~m}, 5 \mathrm{H}) .{ }^{13} \mathrm{C}-\mathrm{NMR}(75$ $\left.\mathrm{MHz}, \mathrm{CDCl}_{3}\right): \delta=23.2,36.2,53.1,57.2,71.9,78.4,122.5,126.3,127.6,128.5,135.1,136.8$, 170.3, 174.5. GC-MS m/z $295.2(\mathrm{M}+\mathrm{Na})^{+}$. HRMS calculated for $\mathrm{C}_{16} \mathrm{H}_{17} \mathrm{O}_{4}, 273.1121$; found, 273.1124 .

2-(Methoxycarbonyl)-2-(prop-2-ynyl)hex-4-enoic acid (E/Z: 80/20) (3c). ${ }^{1} \mathrm{H}-\mathrm{NMR}(300 \mathrm{MHz}$, $\left.\mathrm{CDCl}_{3}\right): \delta=1.66(\mathrm{~d}, J=6.5 \mathrm{~Hz}, 6 \mathrm{H}), 2.03(\mathrm{t}, J=2.6 \mathrm{~Hz}, 2 \mathrm{H}), 2.71-2.83(\mathrm{~m}, 8 \mathrm{H}), 3.78(\mathrm{~s}, 3 \mathrm{H})$, $3.79(\mathrm{~s}, 3 \mathrm{H}), 5.10-5.25(\mathrm{~m}, 2 \mathrm{H}), 5.55-5.75(\mathrm{~m}, 2 \mathrm{H}) .{ }^{13} \mathrm{C}-\mathrm{NMR}\left(75 \mathrm{MHz}, \mathrm{CDCl}_{3}\right): \delta=17.9,29.5$, $35.3,52.9,57.1,71.6,78.5,123.4,131.0,170.1,175.1$. GC-MS $m / z 233.2(\mathrm{M}+\mathrm{Na})^{+}$. HRMS calculated for $\mathrm{C}_{11} \mathrm{H}_{15} \mathrm{O}_{4}, 211.0971$; found, 211.0978 .

2-(Methoxycarbonyl)-2-(prop-2-ynyl)pent-4-ynoic acid (3d). ${ }^{1} \mathrm{H}-\mathrm{NMR}\left(300 \mathrm{MHz}, \mathrm{CDCl}_{3}\right)$ : $\delta=2.00(\mathrm{t}, J=2.4 \mathrm{~Hz}, 1 \mathrm{H}), 2.94(\mathrm{~d}, J=2.4 \mathrm{~Hz}, 2 \mathrm{H}), 3.74(\mathrm{~s}, 3 \mathrm{H}), 9.87(\mathrm{~s}, 1 \mathrm{H}) .{ }^{13} \mathrm{C}-\mathrm{NMR}(75$ $\left.\mathrm{MHz}_{\mathrm{CDCl}}\right): \delta=22.6,53.4,56.4,72.0,77.9,168.7,173.8$. CI-MS $\left(\mathrm{NH}_{3}\right) \mathrm{m} / z 212\left(\mathrm{M}+\mathrm{NH}_{4}\right)^{+}$. HRMS calculated for $\mathrm{C}_{10} \mathrm{H}_{11} \mathrm{O}_{4}, 195.0657$; found, 195.0654 .

Dimethyl 2-chloro-2-(prop-2-ynyl)malonate (2e). Propargyldimethyl malonate 1 (1.36g; 8 mmol) is placed in a $100 \mathrm{~mL}$ Schlenk tube containing $25 \mathrm{~mL}$ of distilled THF, under argon. The solution is cooled to $0^{\circ} \mathrm{C}$ and $380 \mathrm{mg}$ of a $60 \%$ dispersion of $\mathrm{NaH}$ in oil $(9.6 \mathrm{mmol})$ is added portionwise. Stirring is maintained for $10 \mathrm{~min}$ at $0^{\circ} \mathrm{C}$, then $1.28 \mathrm{~g}$ of $N$-chlorosuccinimide $(9.6$ $\mathrm{mmol}$ ) is added portionwise over $10 \mathrm{~min}$. The reaction is slowly allowed to warm up to RT and the reaction stirred for 16 hours. Solvents are removed under reduced pressure. The solid residue is dissolved in $50 \mathrm{~mL}$ of diethyl ether, and the solution washed twice with $50 \mathrm{~mL}$ of saturated aq. $\mathrm{NH}_{4} \mathrm{Cl}$. The organic layer is collected, dried over $\mathrm{MgSO}_{4}$ and the solvents removed under reduced pressure. The crude yellow oil is purified by column chromatography on silica gel using cyclohexane/ethyl acetate (80/20) as eluent. The product $\mathbf{2 e}$ is obtained as a colorless oil $(1.43 \mathrm{~g}$, 87\%). ${ }^{1} \mathrm{H}-\mathrm{NMR}\left(300 \mathrm{MHz}, \mathrm{CDCl}_{3}\right): \delta=2.12(\mathrm{t}, J=2.6 \mathrm{~Hz}, 1 \mathrm{H}), 3.15(\mathrm{t}, J=2.6 \mathrm{~Hz}, 2 \mathrm{H}), 3.82(\mathrm{~s}$, $6 \mathrm{H}) .{ }^{13} \mathrm{C}-\mathrm{NMR}\left(75 \mathrm{MHz}, \mathrm{CDCl}_{3}\right): \delta=29.3,54.1,68.0,72.5,76.6,166.0 . \mathrm{CI}-\mathrm{MS}\left(\mathrm{NH}_{3}\right) \mathrm{m} / z 222$ $\left(\mathrm{M}+\mathrm{NH}_{4}\right)^{+}, 205(\mathrm{M}+\mathrm{H})^{+}$. HRMS calculated for $\mathrm{C}_{8} \mathrm{H}_{10} \mathrm{O}_{4}{ }^{35} \mathrm{Cl}, 205.0268$; found, 205.0267. Calculated for $\mathrm{C}_{8} \mathrm{H}_{10} \mathrm{O}_{4}{ }^{37} \mathrm{Cl}$, 207.0218; found, 207.0243.

2-Chloro-2-(methoxycarbonyl)pent-4-ynoic acid (3e). ${ }^{1} \mathrm{H}-\mathrm{NMR}\left(300 \mathrm{MHz}, \mathrm{CDCl}_{3}\right): \delta=2.17$ $(\mathrm{t}, J=2.7 \mathrm{~Hz}, 1 \mathrm{H}), 3.20(\mathrm{~d}, J=2.7 \mathrm{~Hz}, 2 \mathrm{H}), 3.89(\mathrm{~s}, 3 \mathrm{H}), 7.16$ (br. s, $1 \mathrm{H}) .{ }^{13} \mathrm{C}-\mathrm{NMR}(75 \mathrm{MHz}$, $\left.\mathrm{CDCl}_{3}\right): \delta=29.1,54.4,67.8,72.8,76.4,166.0,169.3$. CI-MS $\left(\mathrm{NH}_{3}\right) \mathrm{m} / z 208\left(\mathrm{M}+\mathrm{NH}_{4}\right)^{+}$. HRMS calculated for $\mathrm{C}_{7} \mathrm{H}_{7} \mathrm{O}_{4}{ }^{35} \mathrm{Cl}, 191.0111$; found, 191.0114 .

6-Hydroxy-2-(methoxycarbonyl)-2-(prop-2-ynyl)hex-4-enoic acid (3f) (E/Z: 1/1). ${ }^{1} \mathrm{H}-\mathrm{NMR}$ $\left(300 \mathrm{MHz}, \mathrm{CDCl}_{3}\right): \delta=2.05(\mathrm{t}, J=2.7 \mathrm{~Hz}, 1 \mathrm{H}), 2.08(\mathrm{t}, J=2.7 \mathrm{~Hz}, 1 \mathrm{H}), 2.78-2.89(\mathrm{~m}, 8 \mathrm{H}), 3.79$ (s, 3H), $3.80(\mathrm{~s}, 3 \mathrm{H}), 4.10(\mathrm{~d}, J=5.5 \mathrm{~Hz}, 2 \mathrm{H}), 4.22(\mathrm{~d}, J=7.1 \mathrm{~Hz}, 2 \mathrm{H}), 5.39-5.46(\mathrm{~m}, 2 \mathrm{H}), 5.78-$ $5.90(\mathrm{~m}, 2 \mathrm{H}) .{ }^{13} \mathrm{C}-\mathrm{NMR}\left(75 \mathrm{MHz}, \mathrm{CDCl}_{3}\right): \delta=31.5,34.5,34.8,36.7,53.4,53.5,55.0,58.2$, 62.9, 71.8, 76.6, 124.1, 124.2, 134.1, 135.5, 169.2, 169.3, 172.1, 172.3. CI-MS $\left(\mathrm{NH}_{3}\right) \mathrm{m} / z 244$ $\left(\mathrm{M}+\mathrm{NH}_{4}\right)^{+}, 227(\mathrm{M}+\mathrm{H})^{+}$. HRMS calculated for $\mathrm{C}_{11} \mathrm{H}_{18} \mathrm{O}_{5} \mathrm{~N}, 244.1185$; found, 244.1191. 
2-Benzyl-2-(ethoxycarbonyl)pent-4-ynoic acid (3g). ${ }^{1} \mathrm{H}-\mathrm{NMR}\left(300 \mathrm{MHz}, \mathrm{CDCl}_{3}\right): \delta=1.30(\mathrm{t}$, $J=7.1 \mathrm{~Hz}, 3 \mathrm{H}), 2.17(\mathrm{t}, J=2.6 \mathrm{~Hz}, 1 \mathrm{H}), 2.68(\mathrm{dd}, J=17.1 \mathrm{~Hz}, 2.7 \mathrm{~Hz}, 1 \mathrm{H}), 2.76(\mathrm{dd}, J=17.1$ $\mathrm{Hz}, 2.7 \mathrm{~Hz}, 1 \mathrm{H}), 3.39$ (q, $J=13.8 \mathrm{~Hz}, 2 \mathrm{H}), 4.23$ (dd, $J=7.1 \mathrm{~Hz}, 1.5 \mathrm{~Hz}, 1 \mathrm{H}), 4.28$ (dd, $J=7.1$ $\mathrm{Hz}, 1.5 \mathrm{~Hz}, 1 \mathrm{H}), 7.18-7.30(\mathrm{~m}, 5 \mathrm{H}) .{ }^{13} \mathrm{C}-\mathrm{NMR}\left(75 \mathrm{MHz}, \mathrm{CDCl}_{3}\right): \delta=14.1,22.4,37.8,40.3$, 59.3, 71.3, 82.7, 125.7, 127.6, 129.8, 139.0, 169.2, 173.0. CI-MS $\left(\mathrm{NH}_{3}\right) m / z 278\left(\mathrm{M}+\mathrm{NH}_{4}\right)^{+}, 261$ $(\mathrm{M}+\mathrm{H})^{+}$. HRMS calculated for $\mathrm{C}_{15} \mathrm{H}_{17} \mathrm{O}_{4}, 261.1127$; found, 261.1134.

2-(Ethoxycarbonyl)-2-(prop-2-ynyl)hexanoic acid (3h). ${ }^{1} \mathrm{H}-\mathrm{NMR}\left(300 \mathrm{MHz}, \mathrm{CDCl}_{3}\right): \delta=0.90$ $(\mathrm{t}, J=7.2 \mathrm{~Hz}, 3 \mathrm{H}), 1.10-1.40(\mathrm{~m}, 7 \mathrm{H}), 2.00-2.10(\mathrm{~m}, 3 \mathrm{H}), 2.80(\mathrm{dd}, J=17.1 \mathrm{~Hz}, 2.7 \mathrm{~Hz}, 1 \mathrm{H})$, 2.90 (dd, $J=17.1 \mathrm{~Hz}, 2.7 \mathrm{~Hz}, 1 \mathrm{H}), 4.29$ (q, $J=7.2 \mathrm{~Hz}, 1 \mathrm{H}), 10.3$ (br. s, $1 \mathrm{H}) .{ }^{13} \mathrm{C}-\mathrm{NMR}(75 \mathrm{MHz}$, $\left.\mathrm{CDCl}_{3}\right): \delta=13.8,13.9,22.7,23.2,26.2,32.4,57.0,62.1,71.5,78.6,170.7,175.7 . \mathrm{CI}-\mathrm{MS}\left(\mathrm{NH}_{3}\right)$ $m / z 244\left(\mathrm{M}+\mathrm{NH}_{4}\right)^{+}, 227(\mathrm{M}+\mathrm{H})^{+}$. HRMS calculated for $\mathrm{C}_{12} \mathrm{H}_{19} \mathrm{O}_{4}, 227.1283$; found, 227.1282.

Diethyl 2-butyl-2-(3-phenylprop-2-ynyl)malonate (2i). ${ }^{1} \mathrm{H}-\mathrm{NMR}\left(300 \mathrm{MHz}, \mathrm{CDCl}_{3}\right), \delta=0.91$ $(\mathrm{t}, J=7.2 \mathrm{~Hz}, 3 \mathrm{H}), 1.15-1.42(\mathrm{~m}, 10 \mathrm{H}), 2.00-2.15(\mathrm{~m}, 2 \mathrm{H}), 3.03(\mathrm{~s}, 2 \mathrm{H}), 4.21(\mathrm{q}, J=7.1 \mathrm{~Hz}, 1 \mathrm{H})$, 7.25-7.37 (m, 5H). ${ }^{13} \mathrm{C}-\mathrm{NMR}\left(75 \mathrm{MHz}, \mathrm{CDCl}_{3}\right), \delta=14.7,14.8,23.6,24.3,26.8,32.4,57.9,62.2$, 83.9, 85.3, 124.0, 128.7, 128.8, 132.3, 171.2. CI-MS $\left(\mathrm{NH}_{3}\right) \mathrm{m} / z 348\left(\mathrm{M}+\mathrm{NH}_{4}\right)^{+}, 331(\mathrm{M}+\mathrm{H})^{+}$. HRMS calculated for $\mathrm{C}_{20} \mathrm{H}_{27} \mathrm{O}_{4}, 331.1909$; found, 331.1903 .

2-(Ethoxycarbonyl)-2-(3-phenylprop-2-ynyl)hexanoic acid (3i). ${ }^{1} \mathrm{H}-\mathrm{NMR}\left(300 \mathrm{MHz}, \mathrm{CDCl}_{3}\right)$ : $\delta=0.90(\mathrm{t}, J=7.0 \mathrm{~Hz}, 3 \mathrm{H}), 1.22-1.37(\mathrm{~m}, 7 \mathrm{H}), 2.00-2.12(\mathrm{~m}, 2 \mathrm{H}), 2.98(\mathrm{~d}, J=17.0 \mathrm{~Hz}, 1 \mathrm{H})$, $3.11(\mathrm{~d}, J=17.0 \mathrm{~Hz}, 1 \mathrm{H}), 4.30(\mathrm{q}, J=7.0 \mathrm{~Hz}, 2 \mathrm{H}), 7.26-7.37(\mathrm{~m}, 5 \mathrm{H}) .{ }^{13} \mathrm{C}-\mathrm{NMR}(75 \mathrm{MHz}$, $\left.\mathrm{CDCl}_{3}\right): \delta=12.8,13.0,21.8,23.1,25.2,31.3,56.4,61.0,82.5,83.1,122.1,127.0,127.2,130.6$, 169.7, 175.2. CI-MS $\left(\mathrm{NH}_{3}\right) \mathrm{m} / z 320\left(\mathrm{M}+\mathrm{NH}_{4}\right)^{+}, 303(\mathrm{M}+\mathrm{H})^{+}$. HRMS calculated for $\mathrm{C}_{18} \mathrm{H}_{23} \mathrm{O}_{4}$, 303.1596; found, 303.1594.

Diethyl 2-butyl-2-(3-(4-cyanophenyl)prop-2-ynyl)malonate $\quad(\mathbf{2 j}) .{ }^{1} \mathrm{H}-\mathrm{NMR} \quad(300 \mathrm{MHz}$, $\left.\mathrm{CDCl}_{3}\right): \delta=0.91(\mathrm{t}, J=7.1 \mathrm{~Hz}, 3 \mathrm{H}), 1.22-1.42(\mathrm{~m}, 10 \mathrm{H}), 2.04-2.10(\mathrm{~m}, 2 \mathrm{H}), 3.05(\mathrm{~s}, 2 \mathrm{H}), 4.22$ $(\mathrm{q}, J=7.1 \mathrm{~Hz}, 4 \mathrm{H}), 7.42(\mathrm{~d}, J=8.0 \mathrm{~Hz}, 2 \mathrm{H}), 7.56(\mathrm{~d}, J=8.0 \mathrm{~Hz}, 2 \mathrm{H}) .{ }^{13} \mathrm{C}-\mathrm{NMR}(75 \mathrm{MHz}$, $\left.\mathrm{CDCl}_{3}\right): \delta=13.8,14.1,22.8,23.7,26.1,31.8,57.0,61.6,81.9,111.3,118.5,128.3,131.9,132.2$, 170.3. CI-MS $\left(\mathrm{NH}_{3}\right) \mathrm{m} / z 373\left(\mathrm{M}+\mathrm{NH}_{4}\right)^{+}, 356(\mathrm{M}+\mathrm{H})^{+}$. HRMS calculated for $\mathrm{C}_{21} \mathrm{H}_{26} \mathrm{O}_{4} \mathrm{~N}$, 356.1862; found, 356.1866.

2-(Ethoxycarbonyl)-2-(3-(4-cyanophenyl)prop-2-ynyl)hexanoic acid (3j). ${ }^{1} \mathrm{H}-\mathrm{NMR}$ (300 $\left.\mathrm{MHz}, \mathrm{CDCl}_{3}\right): \delta=0.91(\mathrm{t}, J=7.0 \mathrm{~Hz}, 3 \mathrm{H}), 1.22-1.43(\mathrm{~m}, 7 \mathrm{H}), 2.03-2.10(\mathrm{~m}, 2 \mathrm{H}), 3.03(\mathrm{~d}, J=$ $17.3 \mathrm{~Hz}, 1 \mathrm{H}), 3.12$ (d, $J=17.3 \mathrm{~Hz}, 1 \mathrm{H}), 4.28$ (q, $J=7.0 \mathrm{~Hz}, 2 \mathrm{H}), 7.42$ (d, $J=8.0 \mathrm{~Hz}, 2 \mathrm{H}), 7.56$ $(\mathrm{d}, J=8.0 \mathrm{~Hz}, 2 \mathrm{H}) .{ }^{13} \mathrm{C}-\mathrm{NMR}\left(75 \mathrm{MHz}, \mathrm{CDCl}_{3}\right): \delta=13.8,14.0,22.7,24.8,26.4,33.3,57.2$, $62.3,82.2,89.1,111.5,118.4,128.0,131.9,132.2,171.5,174.1$. CI-MS $\left(\mathrm{NH}_{3}\right) \mathrm{m} / z 345$ $\left(\mathrm{M}+\mathrm{NH}_{4}\right)^{+}, 328(\mathrm{M}+\mathrm{H})^{+}$. HRMS calculated for $\mathrm{C}_{19} \mathrm{H}_{22} \mathrm{O}_{4} \mathrm{~N}, 328.1549$; found, 328.1547.

Diethyl 2-butyl-2-(3-(4-methoxyphenyl)prop-2-ynyl)malonate (2k). ${ }^{1} \mathrm{H}-\mathrm{NMR}(300 \mathrm{MHz}$, $\left.\mathrm{CDCl}_{3}\right): \delta=0.91(\mathrm{t}, J=7.1 \mathrm{~Hz}, 3 \mathrm{H}), 1.94-2.06(\mathrm{~m}, 10 \mathrm{H}), 2.10-2.12(\mathrm{~m}, 2 \mathrm{H}), 3.00(\mathrm{~s}, 2 \mathrm{H}), 3.78$ $(\mathrm{s}, 3 \mathrm{H}), 4.20(\mathrm{q}, J=8.0 \mathrm{~Hz}, 4 \mathrm{H}), 6.79(\mathrm{~d}, J=8.9 \mathrm{~Hz}, 2 \mathrm{H}), 7.28(\mathrm{~d}, J=8.9 \mathrm{~Hz}, 2 \mathrm{H}) .{ }^{13} \mathrm{C}-\mathrm{NMR}(75$ $\left.\mathrm{MHz}, \mathrm{CDCl}_{3}\right): \delta=13.9,14.1,22.9,23.6,26.1,31.7,55.2,57.2,61.4,77.2,83.0,113.8,115.5$, 133.0, 159.3, 170.6. CI-MS $\left(\mathrm{NH}_{3}\right) \mathrm{m} / z 378\left(\mathrm{M}+\mathrm{NH}_{4}\right)^{+}, 361(\mathrm{M}+\mathrm{H})^{+}$. HRMS calculated for $\mathrm{C}_{21} \mathrm{H}_{29} \mathrm{O}_{5}, 361.2015$; found, 361.2012. 
2-(Ethoxycarbonyl)-2-(3-(4-methoxyphenyl)prop-2-ynyl)hexanoic acid (3k). ${ }^{1} \mathrm{H}-\mathrm{NMR}$ (300 $\left.\mathrm{MHz}, \mathrm{CDCl}_{3}\right): \delta=0.90(\mathrm{t}, J=7.0 \mathrm{~Hz}, 3 \mathrm{H}), 1.22-1.37(\mathrm{~m}, 7 \mathrm{H}), 2.00-2.10(\mathrm{~m}, 2 \mathrm{H}), 2.97(\mathrm{~d}, J=$ $17.0 \mathrm{~Hz}, 1 \mathrm{H}), 3.07$ (d, $J=17.0 \mathrm{~Hz}, 1 \mathrm{H}), 3.79$ (s, 3H), 4.27 (q, $J=7.0 \mathrm{~Hz}, 2 \mathrm{H}), 6.79$ (d, $J=8.9$ $\mathrm{Hz}, 2 \mathrm{H}), 7.28(\mathrm{~d}, J=8.9 \mathrm{~Hz}, 2 \mathrm{H}) .{ }^{13} \mathrm{C}-\mathrm{NMR}\left(75 \mathrm{MHz}, \mathrm{CDCl}_{3}\right): \delta=13.8\left(\mathrm{CH}_{3}\right), 14.0\left(\mathrm{CH}_{3}\right), 22.8$ $\left(\mathrm{CH}_{2}\right), 24.8\left(\mathrm{CH}_{2}\right), 26.4\left(\mathrm{CH}_{2}\right), 33.1\left(\mathrm{CH}_{2}\right), 55.3\left(\mathrm{CH}_{2}\right), 57.4\left(\mathrm{CH}_{2}\right), 62.2\left(\mathrm{CH}_{3}\right), 82.4(\mathrm{C}), 83.4$ (C), 113.8 (2 CH Ar.), 115.3 (C), 133.0 (2 CH Ar.), 159.4 (C), 171.8 (C), 175.1 (C). CI-MS $\left(\mathrm{NH}_{3}\right) \mathrm{m} / \mathrm{z} 350\left(\mathrm{M}+\mathrm{NH}_{4}\right)^{+}, 333(\mathrm{M}+\mathrm{H})^{+}$. HRMS calculated for $\mathrm{C}_{19} \mathrm{H}_{25} \mathrm{O}_{5}, 333.1702$; found, 333.1700 .

3-Methoxycarbonyl-5-methylene- $\gamma$-butyrolactone (6k). ${ }^{1} \mathrm{H}-\mathrm{NMR}\left(300 \mathrm{MHz}, \mathrm{CDCl}_{3}\right): \delta=0.86$ $(\mathrm{t}, J=6.8 \mathrm{~Hz}, 3 \mathrm{H}), 1.10-1.30(\mathrm{~m}, 7 \mathrm{H}), 1.80-2.05(\mathrm{~m}, 2 \mathrm{H}), 2.25-2.35(\mathrm{~m}, 2 \mathrm{H}), 2.75-3.10(\mathrm{~m}, 2 \mathrm{H})$, $3.84(\mathrm{~s}, 3 \mathrm{H}), 4.22(\mathrm{q}, J=7.0 \mathrm{~Hz}, 2 \mathrm{H}), 6.89(\mathrm{~d}, J=11.7 \mathrm{~Hz}, 2 \mathrm{H}), 7.90(\mathrm{~d}, J=11.7 \mathrm{~Hz}, 2 \mathrm{H}) .{ }^{13} \mathrm{C}-$ NMR $\left(75 \mathrm{MHz}, \mathrm{CDCl}_{3}\right): \delta=13.7,14.0,22.8,26.9,29.8,33.9,35.7,55.4,57.2,62.1,77.2$, 113.7, 129.6, 130.4, 163.6, 174.7, 197.6. CI-MS $\left(\mathrm{NH}_{3}\right) \mathrm{m} / z 368\left(\mathrm{M}+\mathrm{NH}_{4}\right)^{+}, 351(\mathrm{M}+\mathrm{H})^{+}$. HRMS calculated for $\mathrm{C}_{19} \mathrm{H}_{27} \mathrm{O}_{6}, 351.1812$; found, 351.1808 .

\section{Acknowledgements}

This work was supported by the Centre National de la Recherche Scientifique and partially by a grant from CPER (action 10040 "Pole Chimie du vivant"). E.G. is grateful to the Ministère de l'Education et de la Recherche for financial support (2003-2006). The authors thank M.-N. Rager (Ecole Nationale Supérieure de Chimie de Paris) for NMR NOESY experiments.

\section{References and footnotes}

1. For representative reviews, see: (a) Hashmi, A. S. K. Angew. Chem. Int. Ed. 2005, 44, 6990. (b) Höffmann-Röder, A.; Krause, N. Org. Biomol. Chem. 2005, 3, 387. (c) Hashmi, A. S. K. Gold Bull. 2004, 37, 51. (d) Arcadi, A.; Di Giuseppe, S. Curr. Org. Chem. 2004, 8, 795. (e) Bianchi, G.; Arcadi, A. In Targets in Heterocyclic Systems 2004, 8, 82. (f) Hashmi, A. S. K. Gold Bull. 2003, 36, 3. (g) Dyker, G. Angew. Chem. Int. Ed. 2000, 39, 4237.

2. Georgy, M.; Boucard, V.; Campagne, J.-M. J. Am. Chem. Soc. 2005, 127, 14180. (b) Zhang, L.; Kozmin, S. A. J. Am. Chem. Soc. 2005, 127, 6962. (c) Gagosz, F. Org. Lett. 2005, 7, 4129. (d) Nieto-Oberhuber, C.; Lopez, S.; Echavarren, A. M. J. Am. Chem. Soc. 2005, 127, 6178. (e) Zhang, L.; Kozmin, S. A. J. Am. Chem. Soc. 2004, 126, 11806. (f) Luzung, M. R.; Markham, J. P.; Toste, F. D. J. Am. Chem. Soc. 2004, 126, 10858. (g) Sherry, B. D.; Toste, F. D. J. Am. Chem. Soc. 2004, 126, 15978. (h) Mamane, V.; Gress, T.; Krause, H.; Fürstner, A. J. Am. Chem. Soc. 2004, 126, 8654. (i) Nieto-Oberhuber, C.; Muñoz, M. P.; Bunuel, E.; Nevado, C.; Cardenas, D. J.; Echavarren, A. M. Angew. Chem. Int. Ed. 2004, 43, 2402. (j) Nevado, C.; Cardenas, D. J.; Echavarren, A. M. Chem. Eur. J. 2003, 9, 2627. (k) Hashmi, A 
.S. K.; Frost, T. M.: Bats, J. W. J. Am. Chem. Soc. 2000, 122, 11553. (1) Reich, N. W.; Yang, C.-G.; Shi, Z.; He, C. Synlett 2006, 1278. (m) Sherry, B.D.; Maus, L.; Laforteza, B.N.; Toste, F.D. J. Am. Chem. Soc. 2006, 128, 8132. (o) Nieto-Oberhuber, C.; Muñoz, M.P.; Lopezl, S.; Jimenez-Nunez, E.; Nevado, C.; Herrero-Gomez, E.; Raducan, M.; Echavarren, A.M. Chem. Eur. J. 2006, 12, 1677. (p) Marion, N.; de Frémont, P.; Lemière, G.; Stevens, E.D.; Fensterbank, L.; Malacria, M.; Nolan, S.P. Chem. Commun. 2006, 2048.

3. Morita, N.; Krause, N. Angew. Chem. Int. Ed. Engl. 2006, 45, 1897. (b) Nakamura, I.; Sato, T.; Yamamoto, Y. Angew. Chem. Int. Ed. Engl. 2006, 45, 4473.

4. Shi, X.; Gorin, D.J.; Toste, F.D. J. Am. Chem. Soc. 2005, 127, 5802. (b) Suhre, M.H.; Reif, M.; Kirsch, S.F. Org. Lett. 2005, 7, 3925. (c) Yao, T.; Zhang, X.; Larock, R.C. J. Am. Chem. Soc. 2004, 126, 11164. (d) Hashmi, A.S.K.; Weyrauch, J.P.; Frey, W.; Bats, J.W. Org. Lett. 2004, 6, 4391. (e) Asao, N.; Nogami, T.; Lee, S.; Yamamoto, Y. J. Am. Chem. Soc. 2003, 126, 7458-7459. (f) Gasparrini, F.; Giovannoli, M.; Misiti, D.; Natile, G.; Palmieri, G.; Maresca, L. J. Am. Chem. Soc. 1993, 115, 4401. (g) Hoffmann-Roder, A.; Krause, N. Org. Lett. 2001, 3, 2537. (h) Yang, C.-G.; He, C. J. Am. Chem. Soc. 2005, 127, 6966. (i) Zhou, C.-Y.; Chan, P.W.H.; Che, C.-M. Org. Lett. 2006, 8, 325. (j) Yao, X.; Li, C.-J. Org. Lett. 2006, 8, 1953. (k) Buzas, A.; Istrate, F.; Gagosz, F. Org. Lett. 2006, 8, 1957-1959. (1) Jung, H.H.; Floreancig, P.E. Org. Lett. 2006, 8, 1949-1951. (m) Barluenga, J.; Diéguez, A.; Fernandez, A.; Rodriguez, F.; Fananas, F.J. Angew. Chem. Int. Ed. Engl. 2006, 45, 2091. (n) Sromek, A.W.; Rubina, M.; Gevorgyan, V. J. Am. Chem. Soc. 2005, 127, 10500. (o) Liu, Y.; Song, F.; Song, Z.; Liu, M.; Yan, B. Org. Lett. 2005, 7, 5409. (p) Zhu, J.; Germain, A.R.; Porco Jr., J.A. Angew. Chem. Int. Ed. Engl. 2004, 43, 1239. (q) Kang, J.-E.; Lee, E.-S.; Park, S.-I.; Shin, S. Tetrahedron Lett. 2005, 46, 7431. (r) Wang, S.; Zhang, L. J. Am. Chem. Soc. 2006, 128, 9979. (s) Liu, Y. Liu, M.; Guo, S.; Huamin, T.; Zhou, Y.; Gao, H. Org. Lett. 2006, 8, 3445.

5. Gorin, D. J.; Davis, N. R.; Toste, F. D. J. Am. Chem. Soc. 2005, 127, 11260. (b) Alfonsi, M.; Arcadi, A.; Aschi, M.; Bianchi, G.; Marinelli, F. J. Org. Chem. 2005, 70, 2265 and references cited therein. (c) Arcadi, A.; Bianchi, G.; Marinelli, F. Synthesis 2004, 610. (d) Arcadi, A.; Di Giuseppe, S.; Marinelli, F.; Rossi, E. Adv. Synth. Catal. 2001, 343, 443. (e) Arcadi, A.; Di Giuseppe, S.; Marinelli, F.; Rossi, E. Tetrahedron: Asymmetry 2001, 12, 2715. (f) Morita, N. Krause, N. Org. Lett. 2004, 6, 4121. (g) Liu, X.-Y.; Li, C.-H.; Che, C.M. Org. Lett. 2006, 8, 2707. (h) Zhang, J.; Yang, C.-G.; He, C. J. Am. Chem. Soc. 2006, 128, 1798. (i) Han, X.; Widenhoefer, R. A. Angew. Chem. Int. Ed. 2006, 45, 1747. (j) Zhang, Z.; Liu, C.; Kinder, R.; Han, X.; Qian, H.; Widenhoefer, R. A. J. Am. Chem. Soc. 2006, 128, 9066.

6. Genin, E.; Toullec, P. Y.; Antoniotti, S.; Brancour, C.; Genêt, J.-P.; Michelet, V. J. Am. Chem. Soc. 2006, 128, 3112. (b) Genin, E.; Antoniotti, S.; Michelet, V.; Genêt, J.-P. Angew. Chem. Int. Ed. Engl. 2005, 44, 4949. (c) Antoniotti, S.; Genin, E.; Michelet, V.; Genêt, J.-P. J. Am. Chem. Soc. 2005, 127, 9976. (d) Charruault, L.; Michelet, V.; Taras, R.; Gladiali, S.; Genêt, J.-P. Chem. Commun. 2004, 850. (e) Nevado, L. Charruault, V. Michelet, C. Nieto- 
Oberhuber, M. P. Muñoz, M. Méndez, M.-N. Rager, J.-P. Genêt, A. M. Echavarren, Eur. J. Org. Chem. 2003, 706. (f) Michelet, V.; Charruault, L.; Gladiali, S.; Genêt, J.-P. Pure Appl. Chem. 2006, 78, 397. (g) Toullec, P. Y.; Genin, E.; Leseurre, L.; Genêt, J.-P.; Michelet, V. Angew. Chem. Int. Ed.. 2006, 45, in press.

7. For the intermolecular addition of carboxylic acids to alkenes, see: Yang, C.-G.; He, C. J. Am. Chem. Soc. 2005, 127, 6966.

8. For recent reviews, see: (a) Alonso, F.; Beletskaya, I. P.; Yus, M. Chem. Rev. 2004, 104, 3079. (b) Nakamura, I.; Yamamoto, Y. Chem. Rev. 2004, 104, 2127. (c) Beller, M.; Seayad, J.; Tillack, A.; Jiao, H. Angew. Chem. Int. Ed. Engl. 2004, 43, 3368.

9. Carter, N. B.; Nadany, A. E.; Sweeny, J. B. J. Chem. Soc., Perkin Trans 1 2002, 2324. (b) Collins, I. J. Chem. Soc. Perkin Trans 1 2000, 2845. (c) Negishi, E.-I.; Korota, M. Tetrahedron 1997, 53, 6707. (d) For representative examples from Pd chemistry, see: (a) Balme, G.; Monteiro, N.; Bouyssi, D. Handbook of Organopalladium Chemistry for Organic Synthesis (Ed. E.-I. Negishi) John Wiley \& Sons, 2002, p 2245. (b) Hosokawa, T.; Murahashi, S.-I. Handbook of Organopalladium Chemistry for Organic Synthesis (Ed. E.-I. Negishi) John Wiley \& Sons, 2002, p 2169. (c) Xu, C.; Negishi, E.-I Handbook of Organopalladium Chemistry for Organic Synthesis (Ed. E.-I. Negishi) John Wiley \& Sons, 2002, p 2289.

10. Rahman, A.-U.; Beisler, J. A.; Harley-Mason, J. Tetrahedron 1980, 36, 1063.

11. Trost, B. M.; Lee, D. C. J. Org. Chem. 1989, 54, 2271.

12. Carter, N. B.; Nadany, A.E.; Sweeny, J. B. J. Chem. Soc. Perkin Trans 1 2002, 2324. (b) Collins, I. J. Chem. Soc. Perkin Trans 1 2000, 2845. (c) Negishi, E.-I.; Korota, M. Tetrahedron 1997, 53, 6707. (d) For representative examples from Pd chemistry, see: (a) Balme, G.; Monteiro, N.; Bouyssi, D. Handbook of Organopalladium Chemistry for Organic Synthesis (Ed. E.-I. Negishi) John Wiley \& Sons, 2002, p 2245. (b) Hosokawa, T.; Murahashi, S.-I. Handbook of Organopalladium Chemistry for Organic Synthesis (Ed. E.-I. Negishi) John Wiley \& Sons, 2002, p 2169. (c) Xu, C.; Negishi, E.-I Handbook of Organopalladium Chemistry for Organic Synthesis (Ed. E.-I. Negishi) John Wiley \& Sons, 2002, p 2289.

13. Grissom, J. W.; Klingberg, D.; Huang, D.; Slattery, B. J. J. Org. Chem. 1997, 62, 603.

14. For recent reviews, see: (a) Alonso, F.; Beletskaya, I. P.; Yus, M. Chem. Rev. 2004, 104, 3079. (b) Nakamura, I.; Yamamoto, Y. Chem. Rev. 2004, 104, 2127. (c) Beller, M.; Seayad, J.; Tillack, A.; Jiao, H. Angew. Chem., Int. Ed. Engl. 2004, 43, 3368.

15. Trost, B. M.; Shi, Y. J. Am. Chem. Soc. 1993, 115, 12491.

16. Trost, B. M.; Toste, F. D. J. Am. Chem. Soc. 2002, 124, 5025.

17. Llerena, D.; Aubert, C.; Malacria, M. Tetrahedron Lett. 1996, 37, 7027.

18. Wu, Z.; Minhas, G. S.; Wen, D.; Jiang, H.; Chen, K.; Zimniak, P.; Zheng, J. J. Med. Chem. 2004, 47, 3282.

19. Beaufils, F.; Denes, F.; Renaud, P. Org. Lett. 2004, 6, 2563. 